\title{
ON RIGHT DERIVATIONS OF INCLINE ALGEBRAS
}

\author{
KYUNG Ho KIM*
}

\begin{abstract}
In this paper, we introduce the concept of a right derivation in incline algebras and give some properties of incline algebras. Also, the concept of $d$-ideal is introduced in an incline algebra with respect to right derivation.
\end{abstract}

\section{Introduction}

Z. Q. Cao, K. H. Kim and F. W. Roush [4] introduced the notion of incline algebras in their book and later it was developed by some authors $[1,2,3,5]$. Ahn et al [1] introduced the notion of quotient incline and obtained the structure of incline algebras. N. O. Alshehri [3] introduced the notion of derivation in incline algebra. Incline algebra is a generalization of both Boolean and fuzzy algebra and it is a special type of semiring. It has both a semiring structure and a poset structure. It can also be used to represent automata and other mathematical systems, to study inequalities for non-negative matrices of polynomials. In this paper, we introduce the concept of a right derivation in incline algebras and give some properties of incline algebras. Also, the concept of $d$-ideal is introduced in an incline algebra with respect to right derivation.

\section{Preliminaries}

An incline algebra is a set $K$ with two binary operations denoted by "+" and "*" satisfying the following axioms:

(K1) $x+y=y+x$,

(K2) $x+(y+z)=(x+y)+z$,

Received April 11, 2013; Accepted July 08, 2013.

2010 Mathematics Subject Classification: Primary 06F35, 03G25, 08A30.

Key words and phrases: incline algebra, right derivation, distributive lattice, isotone, $d$-ideal, $k$-ideal.

The research was supported by a grant from the Academic Research Program of Korea National University of Transportation in 2013. 
(K3) $x *(y * z)=(x * y) * z$,

(K4) $x *(y+z)=(x * y)+(x * z)$,

(K5) $(y+z) * x=(y * x)+(z * x)$,

(K6) $x+x=x$,

(K7) $x+(x * y)=x$,

(K8) $y+(x * y)=y$,

for all $x, y, z \in K$. For convenience, we pronounce "+" (resp. "*") as addition (resp. multiplication). Every distributive lattice is an incline algebra. An incline algebra is a distributive lattice if and only if $x * x=x$ for all $x \in K$. Note that $x \leq y \Leftrightarrow x+y=y$ for all $x, y \in K$. It is easy to se that " $\leq$ " is a partial order on $K$ and that for any $x, y \in K$, the element $x+y$ is the least upper bound of $\{x, y\}$. We say that $\leq$ is induced by operation + .

In an incline algebra $K$, the following properties hold.

(K9) $x * y \leq x$ and $y * x \leq x$ for all $x, y \in K$,

(K10) $y \leq z$ implies $x * y \leq x * z$ and $y * x \leq z * x$, for all $x, y, z \in K$,

(K11) If $x \leq y$ and $a \leq b$, then $x+a \leq y+b$, and $x * a \leq y * b$ for all $x, y, a, b \in K$.

Furthermore, an incline algebra $K$ is said to be commutative if $x * y=$ $y * x$ for all $x, y \in K$.

A subincline of an incline algebra $K$ is a non-empty subset $M$ of $K$ which is closed under the addition and multiplication. A subincline $M$ is called an ideal if $x \in M$ and $y \leq x$ then $y \in M$. An element " 0 " in an incline algebra $K$ is a zero element if $x+0=x=0+x$ and $x * 0=0=0 * x$ for any $x \in K$. An non-zero element " 1 " is called a multiplicative identity if $x * 1=1 * x=x$ for any $x \in K$. A non-zero element $a \in K$ is said to be a left (resp. right) zero divisor if there exists a non-zero $b \in K$ such hat $a * b=0$ (resp. $b * a=0$ ) A zero divisor is an element of $K$ which is both a left zero divisor and a right zero divisor. An incline algebra $K$ with multiplicative identity 1 and zero element 0 is called an integral incline if it has no zero divisors. By a homomorphism of inclines, we mean a mapping $f$ from an incline algebra $K$ into an incline algebra $L$ such that $f(x+y)=f(x)+f(y)$ and $f(x * y)=f(x) * f(y)$ for all $x, y \in K$.

\section{Right derivations of incline algebras}

In what follows, let $K$ denote an incline algebra with a zero-element unless otherwise specified. 
Definition 3.1. Let $K$ be an incline algebra. By a right derivation of $K$, we mean a self map $d$ of $K$ satisfying the identities

$$
d(x+y)=d(x)+d(y) \text { and } d(x * y)=(d(x) * y)+(d(y) * x)
$$

for all $x, y \in K$.

ExAmple 3.2. Let $K=\{0, a, b, 1\}$ be a set in which "+" and "*" is defined by

\begin{tabular}{c|ccccc|cccc}
+ & 0 & $a$ & $b$ & 1 \\
\hline 0 & 0 & $a$ & $b$ & 1 & $*$ & 0 & $a$ & $b$ & 1 \\
$a$ & $a$ & $a$ & $b$ & 1 & 0 & 0 & 0 & 0 & 0 \\
$b$ & $b$ & $b$ & $b$ & 1 & $a$ & 0 & $a$ & $a$ & $a$ \\
1 & 1 & 1 & 1 & 1 & $b$ & 0 & $a$ & $b$ & $b$ \\
1 & 0 & $a$ & $b$ & 1
\end{tabular}

Then it is easy to check that $(K,+, *)$ is an incline algebra. Define a map $d: K \rightarrow K$ by

$$
d(x)= \begin{cases}a & \text { if } x=a, b, 1 \\ 0 & \text { if } x=0\end{cases}
$$

Then we can see that $d$ is a right derivation of the incline algebra $K$.

Proposition 3.3. Let $K$ be a commutative incline algebra. Then for a fixed $a \in K$, the mapping $d_{a}: K \rightarrow K$ given by $d_{a}(x)=x * a$, for every $x \in K$, is a right derivation of $K$.

Proof. Let $K$ be a commutative incline algebra. Then for a fixed $a \in K$, we have

$$
\begin{aligned}
d_{a}(x * y) & =(x * y) * a=((x * y) * a)+((x * y) * a) \\
& =((x * a) * y)+((y * a) * x)=d_{a}(x) * y+d_{a}(y) * x
\end{aligned}
$$

for all $x, y \in K$. This completes the proof.

Proposition 3.4. Let $K$ be a commutative incline algebra. If $K$ is a distributive lattice, $d_{a}(a)=a$ for each $a \in K$.

Proof. Since $K$ is a distributive lattice, we have $x * x=x$ for all $x \in K$. Hence $d_{a}(a)=a * a=a$.

Proposition 3.5. Let $K$ be a commutative incline algebra and $a, b \in$ $K$. Then $d_{a+b}=d_{a}+d_{b}$.

Proof. Let $K$ be a commutative incline algebra and $a, b \in K$. Then for all $c \in K$, we have

$$
d_{a+b}(c)=c *(a+b)=(c * a)+(c * b)=d_{a}(c)+d_{b}(c)=\left(d_{a}+d_{b}\right)(c) .
$$


Proposition 3.6. Let $d$ be a right derivation of an incline algebra $K$. Then we have $d(0)=0$.

Proof. Let $d$ be a right derivation of an incline algebra. Then we have

$$
d(0)=d(0 * 0)=d(0) * 0+d(0) * 0=0+0=0 .
$$

Proposition 3.7. Let $d$ be a right derivation of an incline algebra $K$. If $K$ is a distributive lattice, then $d(x) \leq x$ for all $x \in K$.

Proof. Let $d$ be a right derivation of $K$ and let $K$ be a distributive lattice. Then

$$
\begin{aligned}
d(x) & =d(x * x)=d(x) * x+d(x) * x \\
& =d(x) * x \leq x
\end{aligned}
$$

from (K9) for all $x \in K$.

Proposition 3.8. Let $K$ be an incline algebra and let $d$ be a right derivation of $K$. Then we have $d(x * y) \leq d(x+y)$ for all $x, y \in K$.

Proof. Let $x, y \in K$. By using (K9), we get $d(x) * y \leq d(x)$ and $d(y) * x \leq d(y)$. Thus we get

$$
d(x * y)=(d(x) * y)+(d(y) * x) \leq d(x)+d(y)=d(x+y) .
$$

Proposition 3.9. Let $K$ be an incline algebra and a distributive lattice. Define $d^{2}(x)=d(d(x))$ for all $x \in K$. If $d^{2}=d$, then $d(x * d(x))=$ $d(x)$ for all $x \in K$.

Proof. Let $K$ be an incline algebra and $x \in K$. Then

$$
\begin{aligned}
d(x * d(x)) & =(d(x) * d(x))+\left(d^{2}(x) * x\right) \\
& =d(x)+(d(x) * x) \\
& =d(x) .
\end{aligned}
$$

Proposition 3.10. Let $K$ be an incline algebra and let $d$ be a right derivation of $K$. Then for all $x, y \in K, d(x * y) \leq d(x)$ and $d(x * y) \leq d(y)$.

Proof. Let $x, y \in K$. Then by using (K7), we obtain

$$
d(x)=d(x+x * y)=d(x)+d(x * y) .
$$

Hence we get $d(x * y) \leq d(x)$. Also, $d(y)=d(y+(x * y))=d(y)+d(x * y)$, and so $d(x * y) \leq d(y)$. 
Definition 3.11. Let $K$ be an incline algebra. A mapping $f$ is isotone if $x \leq y$ implies $f(x) \leq f(y)$ for all $x, y \in K$.

Proposition 3.12. Let $d$ be a right derivation of an incline algebra $K$. Then $d$ is isotone.

Proof. Let $x, y \in K$ be such that $x \leq y$. Then $x+y=y$. Hence we have $d(y)=d(x+y)=d(x)+d(y)$, which implies $d(x) \leq d(y)$. This completes the proof.

Proposition 3.13. A sum of two right derivations of an incline algebra $K$ is again a right derivation of $K$.

Proof. Let $d_{1}$ and $d_{2}$ be two right derivations of $K$ respectively. Then we have for all $a, b \in K$,

$$
\begin{aligned}
\left(d_{1}+d_{2}\right)(a * b) & =d_{1}(a * b)+d_{2}(a * b) \\
& =d_{1}(a) * b+d_{1}(b) * a+d_{2}(a) * b+d_{2}(b) * a \\
& =d_{1}(a) * b+d_{2}(a) * b+d_{1}(b) * a+d_{2}(b) * a \\
& =\left(d_{1}+d_{2}\right)(a) * b+\left(d_{1}+d_{2}\right)(b) * a .
\end{aligned}
$$

Clearly, $\left(d_{1}+d_{2}\right)(a+b)=\left(d_{1}+d_{2}\right)(a)+\left(d_{1}+d_{2}\right)(b)$ for all $a, b \in K$. This completes the proof.

Theorem 3.14. Let $K$ be a commutative incline algebra and let $d_{1}, d_{2}$ be right derivations of $K$, respectively. Define $d_{1} d_{2}(x)=d_{1}\left(d_{2}(x)\right)$ for all $x \in K$. If $d_{1} d_{2}=0$, then $d_{2} d_{1}$ is a right derivation of $K$.

Proof. Let $K$ be a commutative incline algebra and $x, y \in K$. Then we have

$$
\begin{aligned}
0 & =d_{1} d_{2}(x * y)=d_{1}\left(d_{2}(x) * y+d_{2}(y) * x\right) \\
& =d_{1} d_{2}(x) * y+d_{1}(y) * d_{2}(x)+d_{1} d_{2}(y) * x+d_{1}(x) * d_{2}(y) \\
& =d_{1}(y) * d_{2}(x)+d_{1}(x) * d_{2}(y)=d_{2}(x) * d_{1}(y)+d_{2}(y) * d_{1}(x) .
\end{aligned}
$$

Then

$$
\begin{aligned}
d_{2} d_{1}(x * y) & =d_{2}\left(d_{1}(x) * y+d_{1}(y) * x\right) \\
& =d_{2} d_{1}(x) * y+d_{2}(y) * d_{1}(x)+d_{2} d_{1}(y) * x+d_{2}(x) * d_{1}(y) \\
& =d_{2} d_{1}(x) * y+d_{2} d_{1}(y) * x .
\end{aligned}
$$

Finally, for all $x, y \in K$, we get

$$
d_{2} d_{1}(x+y)=d_{2}\left(d_{1}(x)+d_{1}(y)\right)=d_{2} d_{1}(x)+d_{2} d_{1}(y) .
$$

This implies that $d_{2} d_{1}$ is a right derivation of a commutative incline algebra $K$. 
Lemma 3.15. Let $K$ be an incline algebra. If every element a commutes with its right derivation $d(a)$, then we have

$$
d\left(a^{n}\right)=n a^{n-1} d(a) .
$$

Proof. By using induction on $n$, we have the result.

Definition 3.16. Let $K$ be an incline algebra and let $d$ be a nontrivial right derivation of $K$. An ideal $I$ of $K$ is called a $d$-ideal if $d(I)=I$.

Since $d(0)=0$, it can be easily observed that the zero ideal $\{0\}$ is a $d$-ideal of $K$. If $d$ is onto, then $d(K)=K$, which implies $K$ is a $d$-ideal of $K$.

ExAMPLE 3.17. In Example 3.2, let $I=\{0, a\}$. Then $I$ is an ideal of $K$. It can be verified that $d(I)=I$. Therefore, $I$ is an $d$-ideal of $K$.

Lemma 3.18. Let $d$ be a right derivation of $K$ and let $I, J$ be any two $d$-ideals of $K$. Then we have $I \subseteq J$ implies $d(I) \subseteq d(J)$.

Proof. Let $I \subseteq J$ and $x \in d(I)$. Then we have $x=d(y)$ for some $y \in I \subseteq J$. Hence we get $x=d(y) \in d(J)$. Therefore, $d(I) \subseteq d(J)$.

Proposition 3.19. Let $K$ be an incline algebra. Then, a sum of any two $d$-ideals is also a $d$-ideal of $K$.

Proof. Let $I$ and $J$ be $d$-ideals of $K$. Then $I+J=d(I)+d(J)=$ $d(I+J)$. Hence $I+J$ is a $d$-ideal of $K$.

Let $d$ be a right derivation of $K$. Define a set Kerd by

$$
\text { Kerd }:=\{x \in K \mid d(x)=0\}
$$

for all $x \in K$.

Proposition 3.20. Let $d$ be a right derivation of an incline algebra $K$. Then Kerd is a subincline of $K$.

Proof. Let $x, y \in K e r d$. Then $d(x)=0, d(y)=0$ and

$$
\begin{aligned}
d(x * y) & =(d(x) * y)+(d(y) * x) \\
& =(0 * y)+(0 * x) \\
& =0+0=0
\end{aligned}
$$

and

$$
\begin{aligned}
d(x+y) & =d(x)+d(y) \\
& =0+0=0 .
\end{aligned}
$$

Therefore, $x * y, x+y \in K e r d$. This completes the proof. 
Proposition 3.21. Let $d$ be a right derivation of an integral incline algebra $K$. Then Kerd is an ideal of $K$.

Proof. By Proposition 3.20, Kerd is a subincline of $K$. Now let $x \in K$ and $y \in K e r d$ such that $x \leq y$. Then $d(y)=0$ and

$$
0=d(y)=d(y+x * y)=d(y)+d(x * y)=0+d(x * y),
$$

which $d(x * y)=0$. Hence we have

$$
0=d(x * y)=(d(x) * y)+(d(y) * x)=d(x) * y .
$$

Since $K$ has no zero divisors, either $d(x)=0$ or $y=0$. If $d(x)=0$, then $x \in K$ Kerd. If $y=0$, then $x \leq y=0$, i.e., $x=0$, which implies $x \in \operatorname{Kerd}$.

Let $d$ be a right derivation of $K$. Define a set Fix $_{d}(K)$ by

$$
\text { Fix }_{d}(K):=\{x \in K \mid d(x)=x\}
$$

for all $x \in K$.

Proposition 3.22. Let $K$ be a commutative incline algebra and let $d$ be a right derivation. Then Fix $(K)$ is a subincline of $K$.

Proof. Let $x, y \in F_{i x}(K)$. Then we have $d(x)=x$ and $d(y)=y$, and so

$$
\begin{aligned}
d(x * y) & =d(x) * y+d(y) * x=x * y+y * x \\
& =x * y+x * y=x * y .
\end{aligned}
$$

Now

$$
d(x+y)=d(x)+d(y)=x+y,
$$

which implies $x+y, x * y \in F i x_{d}(K)$. This completes the proof.

Definition 3.23. Let $K$ be an incline algebra. An element $a \in K$ is said to be additively left cancellative if for all $a, b \in K, a+b=a+c \Rightarrow$ $b=c$. An element $a \in K$ is said to be additively right cancellative if for all $a, b \in K, b+a=c+a \Rightarrow b=c$. It is said to be additively cancellative if it is both left and right cancellative. If every element of $K$ is additively left cancellative, it is said to be additively left cancellative. If every element of $K$ is additively right cancellative, it is said to be additively right cancellative.

Definition 3.24. A subincline $I$ of an incline algebra $K$ is called a $k$-ideal if $x+y \in I$ and $y \in I$, then $x \in I$.

ExAmple 3.25. In Example 3.2, $I=\{0, a, b\}$ is an $k$-ideal of $K$. 
Theorem 3.26. Let $K$ be a commutative incline algebra and additively right cancellative. If $d$ is a right derivation of $K$, then Fix $(K)$ is a $k$-ideal of $K$.

Proof. By Proposition 3.22, Fix $(K)$ is a subincline of $K$. Let $x+$ $y, y \in$ Fix $_{d}(K)$. Then $d(y)=y$ and $x+y=d(x+y)$. Hence $x+y=$ $d(x+y)=d(x)+d(y)=d(x)+y$, which implies $x \in F i x_{d}(K)$. Hence $F i x_{d}(K)$ is a $k$-ideal of $K$.

Proposition 3.27. Let $K$ be an incline algebra and let $d$ be a right derivation of $K$. Then Kerd is a $k$-ideal of $K$.

Proof. From Proposition 3.20, Kerd is a subincline of $K$. Let $x+y \in$ $K$ and $y \in K e r d$. Then we have $d(x+y)=0$ and $d(y)=0$, and so

$$
0=d(x+y)=d(x)+d(y)=d(x)+0=d(x) .
$$

This implies $x \in$ Kerd.

\section{References}

[1] S. S. Ahn, Y. B. Jun, and H. S. Kim, Ideals and quotients of incline algebras, Commun. Korean Math. Soc. 16 (2001), 573-583.

[2] S. S. Ahn and H. S. Kim, On r-ideals in incline algebras, Commun. Korean Math. Soc. 17 (2002), 229-235.

[3] N. O. Alshehri. On derivations of incline algebras, Scientiae Mathematicae Japonicae online, e-2010 (2010), 199-205.

[4] Z. Q. Cao, K. H. Kim, and F. W. Roush, Incline algebra and applications, John Wiley and Sons, New York, 1984.

[5] W. Yao and S. Han, On ideals, filters, and congruences in inclines, Bull. Korean Math. 46 (2009), no. 3, 591-598.

Department of Mathematics

Korea National University of Transportation

Chungju 380-702, Republic of Korea

E-mail: ghkim@ut.ac.kr 